\title{
Peristaltic Transport of a Magneto Non-Newtonian Fluid through A porous medium in a horizontal finite channel
}

\author{
N. T. El-Dabe ${ }^{1}$, G. Ismail ${ }^{2}$, F. O. Darwesh ${ }^{3}$ \\ ${ }^{I}$ (Mathematics Department, Faculty of Education, Ain Shams University, Cairo, Egypt) \\ ${ }^{2}$ (Mathematics Department, Faculty of Science, Zagazig University, Zagazig, Egypt) \\ ${ }^{3}$ (Higher Technological Institute, $10^{\text {th }}$ of Ramadan City, Egypt)
}

\begin{abstract}
The present investigation consists of an analytic treatment of a peristaltic transport of a magneto non-Newtonian fluid through a porous medium in a finite channel under the assumptions of long wavelength and low Reynolds number. The fluid under consideration is obeying Eyring-Powell model. The velocity field, the pressure gradient, the local wall shear stress and the volume flow rate are obtained in the explicit form. Numerical results are graphically discussed for various values of physical parameters of interest. The results show that the pressure increases as both of the magnetic and the non-Newtonian parameters increasing. The range between peak and trough of local wall shear stress distribution increases with the increasing of the magnetic parameter while this range decreases as the non-Newtonian parameter increases. The reflux region decreases as the magnetic parameter increases but it increases as non-Newtonian parameter increases.
\end{abstract}

Key words: Eyring-Powell Fluid, Magnetic Field, Non-Newtonian, Porous Medium.

\section{Introduction}

Peristalsis is a form of fluid transport induced by a progressive wave of area contraction or expansion along the walls of distensible duct containing a liquid or mixture. A peristaltic pump is a device for pumping fluids, generally from a region of lower to higher pressure, by means of a contraction wave traveling along a tube like structure. Shapiro et al. [1] explained the basic principles and brought out clearly the significances of the various parameters governing the flow. The non-Newtonian effects in peristaltic motion were included in Kaimal [2] .Numerical techniques were used by Takabatake et al. [3] for axisymmetric tube flow. Many authors investigate the effect of magnetic field on peristaltic transport. The effect of the magnetic field on blood flow was studied by Stud et al. [4]. Mekheimer [5] studied the effect of a uniform magnetic field on peristaltic transport of a blood in a non-uniform two-dimensional channel. Afifi [6] studied the aspects of a magneto-fluid with suspended particles. The fluid motion through a porous medium has been studied by many authors [7-15]. Tripathi $[16,17]$ studied the influence of heat transfer in swallowing of food bolus (water) through the oesophagus and heat flow through porous finite channel.

The main aim of this investigation is to generlize the work of Tripathi $[16,17]$ to include the flow of a non-Newtonian fluid which obeying Eyring-Powell model through a porous medium in the presence of a uniform magnetic field without taking heat in our consideration. The relation between the solution of this problem and different parameters are obtained in order to investigate how to control the motion of the fluid by changing these parameters. The numerical discussions of the results are obtained and illustrated graphically through a set of figures.

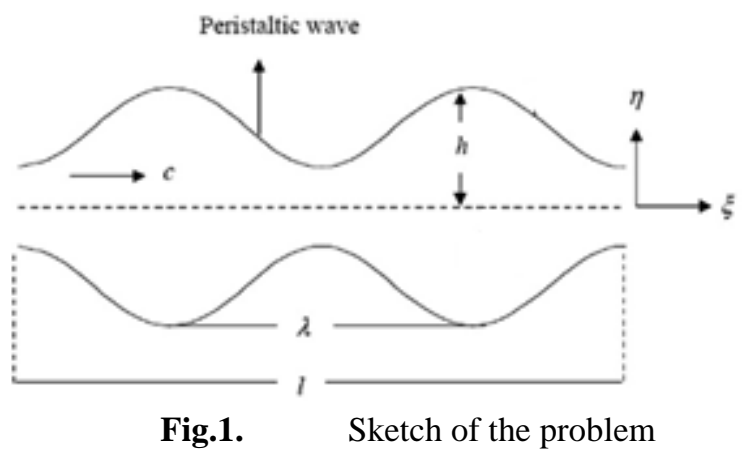




\section{Formulation of the problem}

Choose Cartesian coordinates $\xi, \eta$ where $\xi$ is coincide on the axis of the channel, while $\eta$ is perpendicular to it as seen in figure (1).

The non-Newtonian fluid will flow inside the flexible channel. The system is expressed under the external uniform magnetic field of intensity $B_{0}$ perpendicular to the axis of the channel.

The constitutive equation of the Eyring- Powell model can be written as follows:

$\tilde{\tau}_{i j}=\mu \frac{\partial \tilde{u}_{i}}{\partial \tilde{x}_{j}}+\frac{1}{\varrho} \sinh ^{-1}\left(\frac{1}{\epsilon} \frac{\partial \tilde{u}_{i}}{\partial \tilde{x}_{j}}\right)$

where $\mu$ is the coefficient of viscosity and $\varrho, \epsilon$ are the characteristics of the non-Newtonian Eyring-Powell model.

The constitutive equation of wall geometry due to propagation of train waves is given by

$\tilde{h}(\tilde{\xi}, \tilde{t})=a-\tilde{\phi} \cos ^{2} \frac{\pi}{\lambda}(\tilde{\xi}-c \tilde{t})$

where $\tilde{h}$ is the transverse vibration of the wall, $\tilde{t}$ is the time, $a$ is the half width of the channel, $\tilde{\phi}$ is the amplitude of the wave, $\lambda$ is the wavelength and $c$ is the wave velocity.

The equations governing the motion of an incompressible non-Newtonian fluid with heat transfer are given by

The Continuity Equation:

$\frac{\partial \tilde{u}}{\partial \tilde{\xi}}+\frac{\partial \tilde{v}}{\partial \tilde{\eta}}=0$

The Momentum Equations:

$\rho\left(\frac{\partial}{\partial \tilde{t}}+\tilde{u} \frac{\partial}{\partial \tilde{\xi}}+\tilde{v} \frac{\partial}{\partial \tilde{\eta}}\right) \tilde{u}=-\frac{\partial \tilde{p}}{\partial \tilde{\xi}}+\left(\frac{\partial \tilde{1}_{11}}{\partial \tilde{\xi}}+\frac{\partial \tilde{1}_{12}}{\partial \tilde{\eta}}\right)-\frac{\mu}{k_{0}} \tilde{u}-\sigma B_{0}{ }^{2} \tilde{u}$

$\rho\left(\frac{\partial}{\partial \tilde{t}}+\tilde{u} \frac{\partial}{\partial \tilde{\xi}}+\tilde{v} \frac{\partial}{\partial \tilde{\eta}}\right) \tilde{v}=-\frac{\partial \tilde{p}}{\partial \tilde{\eta}}+\left(\frac{\partial \tilde{\tau}_{21}}{\partial \tilde{\xi}}+\frac{\partial \tilde{\tau}_{22}}{\partial \tilde{\eta}}\right)-\frac{\mu}{k_{0}} \tilde{v}$

where $\rho$ is the fluid density, $\tilde{u}$ is the axial velocity, $\tilde{v}$ is the transverse velocity, $\tilde{p}$ is the pressure, $\sigma$ is the magnetic conductivity of the fluid, $k_{0}$ is the permeability of the porous medium.

Introduce the following non-dimensional parameters

$$
\left.\begin{array}{l}
\xi=\frac{\tilde{\xi}}{\lambda}, \eta=\frac{\tilde{\eta}}{a}, t=\frac{c \tilde{t}}{\lambda}, u=\frac{\tilde{u}}{c}, v=\frac{\tilde{v}}{c \delta}, \delta=\frac{a}{\lambda}, h=\frac{\tilde{h}}{a}, l=\frac{\tilde{l}}{\lambda}, \phi=\frac{\tilde{\phi}}{a}, \\
p=\frac{\tilde{p} a^{2}}{\mu c \lambda}, \psi=\frac{\tilde{\psi}}{a c}, Q=\frac{\tilde{Q}}{a c}, R e=\frac{\rho c a \delta}{\mu}, K=\frac{k_{0}}{a^{2}}, M=\frac{\sigma B_{0}{ }^{2} a^{2}}{\mu}, \Omega=\frac{1}{\mu \sigma \epsilon}
\end{array}\right\}
$$

where $\delta$ is the wave number, $\tilde{l}$ is the characteristic length of the channel, $R e$ is the Reynolds number, $K$ is the permeability parameter, $M$ is the magnetic parameter and $\Omega$ is the non-Newtonian parameter.

Using (6) with the first approximation of Eqn. (1), then the system of equations (3-5) can be written in dimensionless form as follows:

$\frac{\partial u}{\partial \xi}+\frac{\partial v}{\partial \eta}=0$

$\operatorname{Re}\left(\frac{\partial u}{\partial t}+u \frac{\partial u}{\partial \xi}+v \frac{\partial u}{\partial \eta}\right)=-\frac{\partial p}{\partial \xi}+\omega^{2}\left(\delta^{2} \frac{\partial^{2} u}{\partial \xi^{2}}+\frac{\partial^{2} u}{\partial \eta^{2}}\right)-m^{2} u$

$\delta^{2} \operatorname{Re}\left(\frac{\partial v}{\partial t}+u \frac{\partial v}{\partial \xi}+v \frac{\partial v}{\partial \eta}\right)=-\frac{\partial p}{\partial \eta}+\omega^{2}\left(\delta^{4} \frac{\partial^{2} v}{\partial \xi^{2}}+\delta^{2} \frac{\partial^{2} v}{\partial \eta^{2}}\right)$

where the wall equation is:

$h(\xi, t)=1-\frac{\phi}{2}[1+\cos 2 \pi(\xi-t)]$

and

$\omega^{2}=(1+\Omega), \quad m^{2}=\left(\frac{1}{K}+M\right)$ 


\section{Method of solution}

According to the assumption of low Reynolds number and large wavelength approximations, the system of our equations (7-9) can be written as:

$$
\begin{aligned}
& \frac{\partial p}{\partial \xi}=\omega^{2} \frac{\partial^{2} u}{\partial \eta^{2}}-m^{2} u \\
& \frac{\partial p}{\partial \eta}=0 \\
& \frac{\partial u}{\partial \xi}+\frac{\partial v}{\partial \eta}=0
\end{aligned}
$$

With the following appropriate boundary conditions

$$
\left\{\begin{array}{c|c}
\left.\frac{\partial u}{\partial \eta}\right|_{\eta=0}=0 & \begin{array}{c}
\text { the regularity } \\
\text { condition } \\
\text { the no slip } \\
\text { condition } \\
\left.u\right|_{\eta=h}=0
\end{array} \\
\left.v\right|_{\eta=0}=0 & \begin{array}{c}
\text { the absence of } \\
\text { transverse velocity } \\
\text { he transverse vibration } \\
\text { of the wall }
\end{array} \\
\left.v\right|_{\eta=h}=\frac{\partial h}{\partial t} & \begin{array}{c}
\text { and }\left.p\right|_{\xi=l}=p_{l} \\
\text { the finite length condition }
\end{array}
\end{array}\right\}
$$

The solution of Eqn. (12) is:

$u=\varphi_{1}(\xi) \cosh \left(\frac{m}{\omega} \eta\right)+\varphi_{2}(\xi) \sinh \left(\frac{m}{\omega} \eta\right)-\frac{1}{m^{2}} \frac{\partial p}{\partial \xi}$

where the functions $\varphi_{1}(\xi)$ and $\varphi_{2}(\xi)$ can be determined as follows:

$\frac{\partial u}{\partial \eta}=\frac{m}{\omega} \varphi_{1}(\xi) \sinh \left(\frac{m}{\omega} \eta\right)+\frac{m}{\omega} \varphi_{2}(\xi) \cosh \left(\frac{m}{\omega} \eta\right)$

and from the boundary conditions we can obtain the functions $\varphi_{1}(\xi)$ and $\varphi_{2}(\xi)$ as:

$\varphi_{1}(\xi)=\frac{1}{m^{2}} \operatorname{sech}\left(\frac{m}{\omega} h\right) \frac{\partial p}{\partial \xi} \quad, \varphi_{2}(\xi)=0$

Hence, the axial velocity $u$ of the fluid can be written as:

$u=\frac{1}{m^{2}}\left[\cosh \left(\frac{m}{\omega} \eta\right) \operatorname{sech}\left(\frac{m}{\omega} h\right)-1\right] \frac{\partial p}{\partial \xi}$

By using Eqn. (14) with the above result of $u$, we can write the expression of the transverse velocity $v$ as:

$v=\frac{1}{m^{2}} \sinh \left(\frac{m}{\omega} \eta\right) \operatorname{sech}\left(\frac{m}{\omega} h\right) \tanh \left(\frac{m}{\omega} h\right) \frac{\partial h}{\partial \xi} \frac{\partial p}{\partial \xi}-\frac{1}{m^{2}}\left[\left(\frac{\omega}{m}\right) \sinh \left(\frac{m}{\omega} \eta\right) \operatorname{sech}\left(\frac{m}{\omega} h\right)-\eta\right] \frac{\partial^{2} p}{\partial \xi^{2}}$

Now, we can obtain $\frac{\partial h}{\partial t}$ from the conditions (15) as:

$\frac{\partial h}{\partial t}=\frac{1}{m^{2}} \tanh ^{2}\left(\frac{m}{\omega} h\right) \frac{\partial h}{\partial \xi} \frac{\partial p}{\partial \xi}-\frac{1}{m^{2}}\left[\left(\frac{\omega}{m}\right) \tanh \left(\frac{m}{\omega} h\right)-h\right] \frac{\partial^{2} p}{\partial \xi^{2}}$

Since,

$\frac{\partial h}{\partial t}=-\frac{\partial h}{\partial \xi}$

Integrate both sides with respect to $(\xi)$ we have:

$\frac{\omega}{m^{3}}\left[\left(\frac{m}{\omega} h\right)-\tanh \left(\frac{m}{\omega} h\right)\right] \frac{\partial p}{\partial \xi}=A(t)-h$

where $A(t)$ is a constant of integration. 
From Eqn. (23) we can write:

$\frac{\partial p}{\partial \xi}=\left(\frac{m^{3}}{\omega}\right) \frac{A(t)-h}{\left(\frac{m}{\omega} h\right)-\tanh \left(\frac{m}{\omega} h\right)}$

Integrating once again, the pressure difference across the length of channel is obtained as:

$p(\xi, t)-p(\xi, 0)=\left(\frac{m^{3}}{\omega}\right) \int_{0}^{\xi} \frac{A(t)-h}{\left(\frac{m}{\omega} h\right)-\tanh \left(\frac{m}{\omega} h\right)} d \xi$

In the case of $\xi=l$ the constant $A(t)$ can be written as:

$A(t)=\frac{\frac{\omega}{m^{3}}\left(p_{l}-p_{0}\right)+\int_{0}^{l} \frac{h}{\left(\frac{m}{\omega} h\right)-\tanh \left(\frac{m}{\omega} h\right)} d \xi}{\int_{0}^{l} \frac{1}{\left(\frac{m}{\omega} h\right)-\tanh \left(\frac{m}{\omega} h\right)} d \xi}$

Local wall shear stress in dimensionless form is defined as: $\tau_{w}=\left.\frac{\partial u}{\partial \eta}\right|_{\eta=h}$

By using the expression of $u$ from Eqn. (19) it has the following form:

$\tau_{w}=\frac{1}{m \omega} \tanh \left(\frac{m}{\omega} h\right) \frac{\partial p}{\partial \xi}$

From Eq. (24):

$\tau_{w}=\left(\frac{m^{2}}{\omega^{2}}\right) \tanh \left(\frac{m}{\omega} h\right) \frac{A(t)-h}{\left(\frac{m}{\omega} h\right)-\tanh \left(\frac{m}{\omega} h\right)}$

Also, we can calculate the volume flow rate which is defined as: $Q(\xi, t)=\int_{0}^{h} u d \eta$

$Q(\xi, t)=\frac{\omega}{m^{3}}\left[\tanh \left(\frac{m}{\omega} h\right)-\frac{m}{\omega} h\right] \frac{\partial p}{\partial \xi}$

From Eq. (24), we can write the volume flow rate as:

$Q(\xi, t)=h-A(t)$

The relation between the averaged flow rate and flow rate in the wave frame and in laboratory frame is:

$\bar{Q}=q+1-\frac{\phi}{2}=Q-h+1-\frac{\phi}{2}$

Using Eqns. (30) and (31), we can write the relation between the averaged flow rate and the amplitude of the wave as:

$\bar{Q}=1-\frac{\phi}{2}-A(t)$

Using Eqns. (29) and (31), we can write the pressure difference across the length of channel as:

$p(\xi)-p(0)=\int_{0}^{\xi}\left(\frac{m^{3}}{\omega}\right) \frac{\left(\bar{Q}+h-1+\frac{\phi}{2}\right)}{\tanh \left(\frac{m}{\omega} h\right)-\left(\frac{m}{\omega} h\right)} d \xi$

So, we can obtain the pressure difference across one wave length as:

$\Delta p_{1}=p(1)-p(0)=\int_{0}^{1}\left(\frac{m^{3}}{\omega}\right) \frac{\left(\bar{Q}+h-1+\frac{\phi}{2}\right)}{\tanh \left(\frac{m}{\omega} h\right)-\left(\frac{m}{\omega} h\right)} d \xi$

\section{Numerical results and discussion}

In order to estimate the quantitative effects of the various parameters involved in the results of present analysis, Maple software version-15 is used for numerical results and plotting the illustration graphs. The numerical evaluations of the analytical results are obtained for $\Delta p, \tau_{w}$ and $\bar{Q}$ for various parameters through 
Figs. 2-7. To study the pressure distribution along the length of channel, the simplest case known as free pumping is considered, i.e., pressure at the two ends of channel are zero, i.e., $p_{l}=p_{0}=0$.

It is found from Figs.2 (a-d) and Figs.3 (a-d) that the pressure is directed properly with all of the magnetic parameter and the non-Newtonian parameter. Also from Figs. 4 (a-d) and Figs.5 (a-d) we can see that the range between peak and trough of local wall shear stress distribution increases with increasing the magnitude of the magnetic parameter and it decreases with increasing the magnitude of the non-Newtonian parameter. From Figs. 5(a) and 5(b) we can see that the relation between the pressure across one wavelength and averaged flow rate is linear, the figures show that the slope of the line decreases with increasing the magnitude of the magnetic parameter and also with increasing the magnitude of the non-Newtonian parameter. Finally Figs. 6(a) and 6(b) show the relation between the amplitude and the averaged flow rate and we can see that the upper portion of the curve is no reflux region while lower portion is reflux region, It is observed that the area of reflux region decreases with increasing the magnitude of the magnetic parameter and it increases with increasing the magnitude of the non-Newtonian parameter.

\section{Conclusion}

The effect of the uniform magnetic field on the peristaltic motion of a non-Newtonian Eyring-Powell fluid through porous medium in a finite horizontal channel is discussed. This phenomenon is modulated mathematically by a system of differential equations which describe the continuity and momentum. The system is solved analytically under the assumption of long wavelength and low Reynolds number. The velocity, the pressure distribution, the local wall shear stress and the volume flow rate are obtained as functions of the physical parameters of the problem. The effects of these parameters on these distributions are discussed numerically and illustrated graphically. This study has many applications in many scientific fields such as biological, physiological, medical and chemical branches.
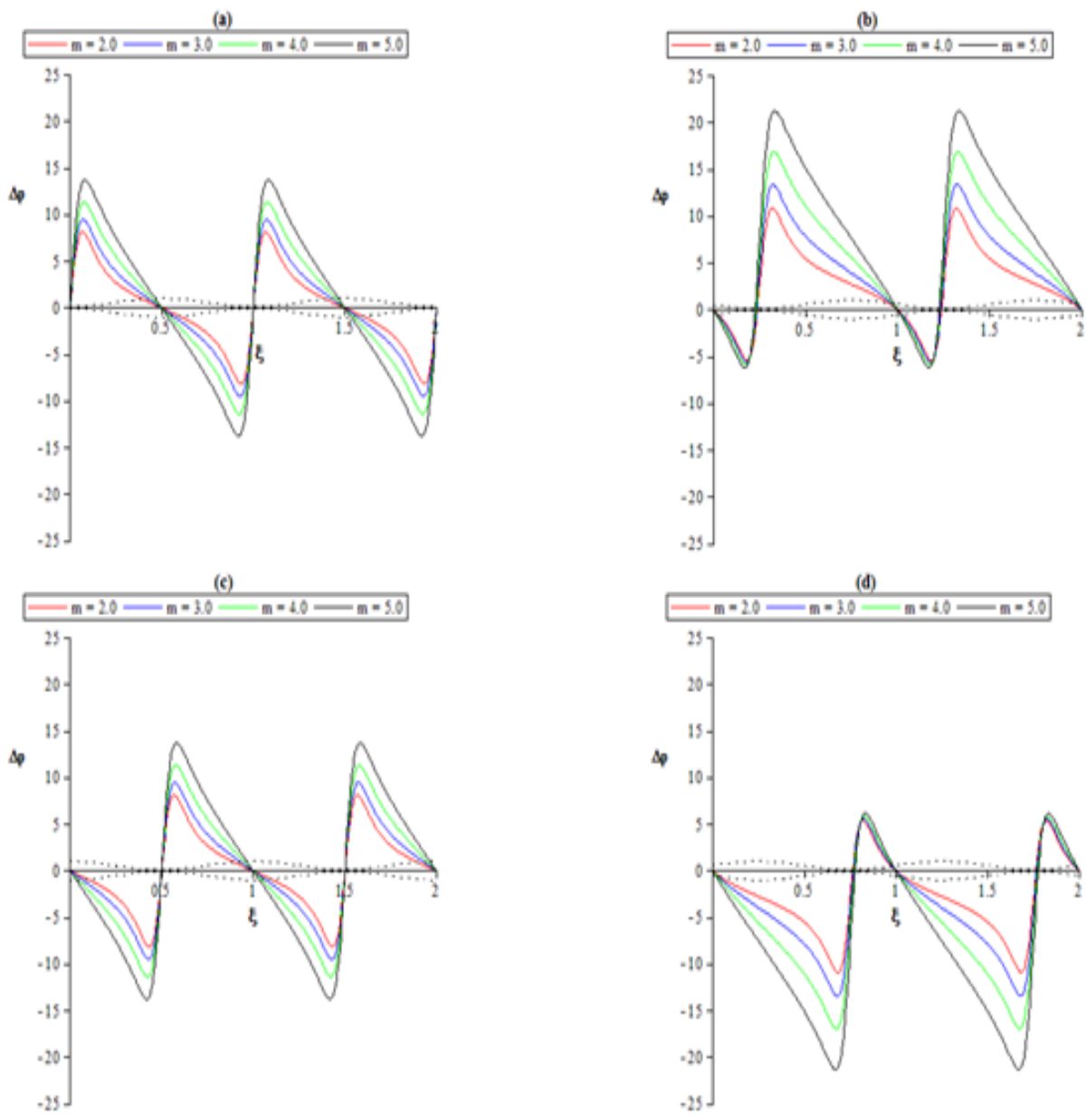

Fig.2. Pressure difference vs. axial distance for $m=2.0,3.0,4.0,5.0, \omega=1.35, \phi=0.9, l=2.0$ and various instants (a) $t=0.0,1.0$ (b) $t=0.25$ (c) $t=0.5$ (d) $t=0.75$. Dotted lines represent the position of wave and color solid lines show pressure along the length of channel. 

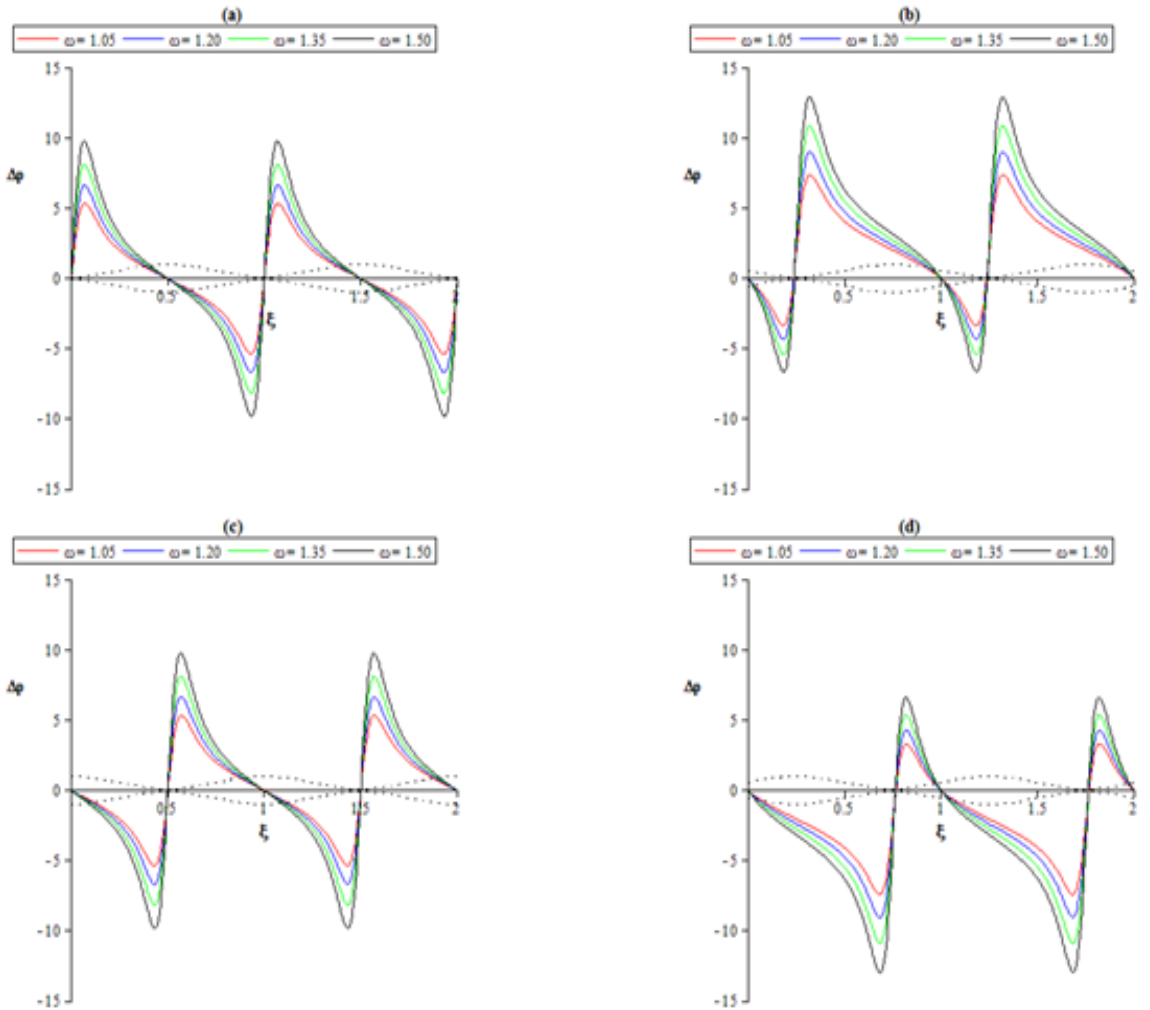

Fig.3. Pressure difference vs. axial distance for $\omega=1.05,1.20,1.35,1.50, m=3.0, \phi=0.9, l=2.0$ and various instants (a) $t=0.0,1.0$ (b) $t=0.25$ (c) $t=0.5$ (d) $t=0.75$. Dotted lines represent the position of wave and color solid lines show pressure along the length of channel.
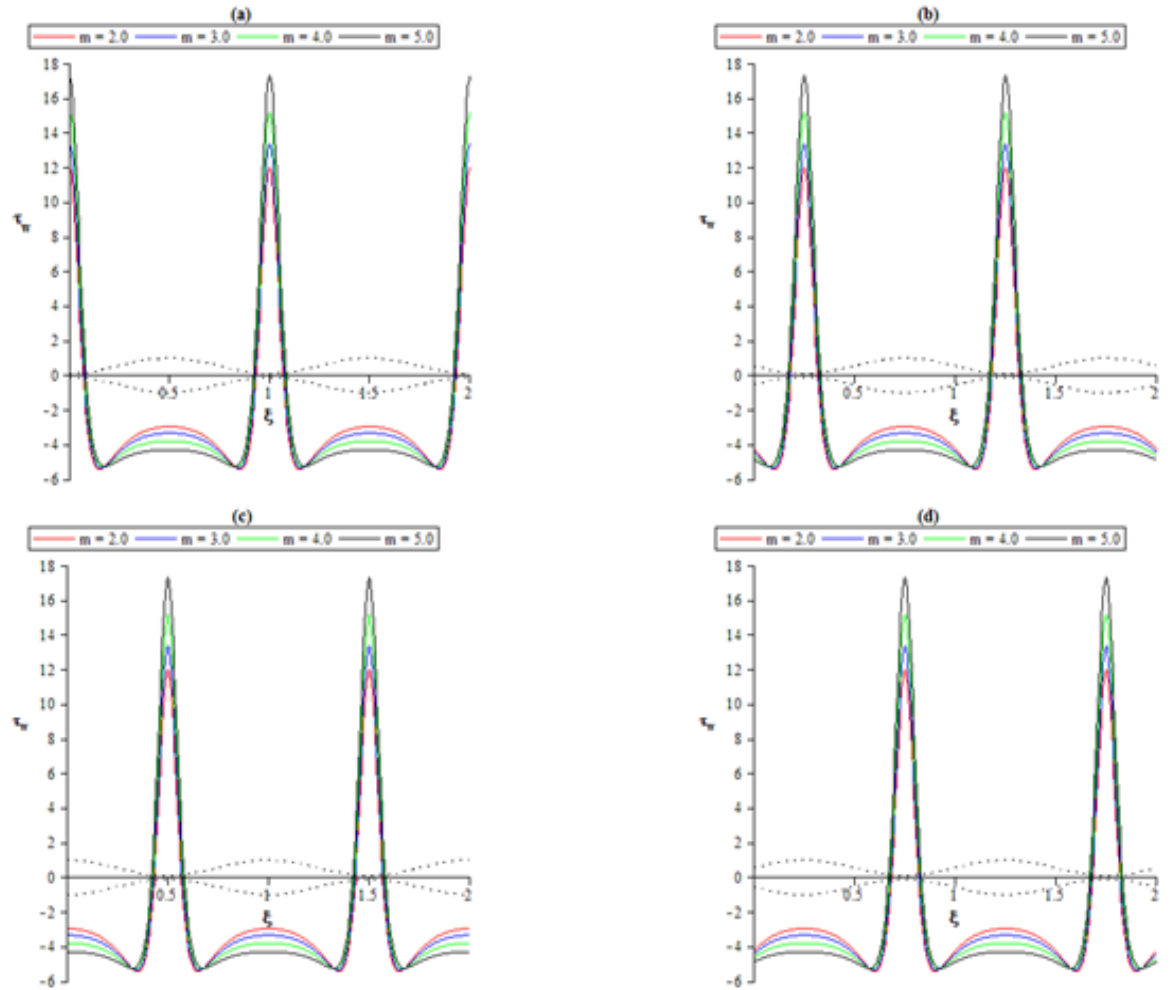

Fig.4. Local wall shear stress vs. axial distance for $m=2.0,3.0,4.0,5.0, \omega=1.35, \phi=0.9, l=2.0$ and various instants (a) $t=0.0,1.0$ (b) $t=0.25$ (c) $t=0.5$ (d) $t=0.75$. Dotted lines represent the position of wave and color solid lines show pressure along the length of channel. 

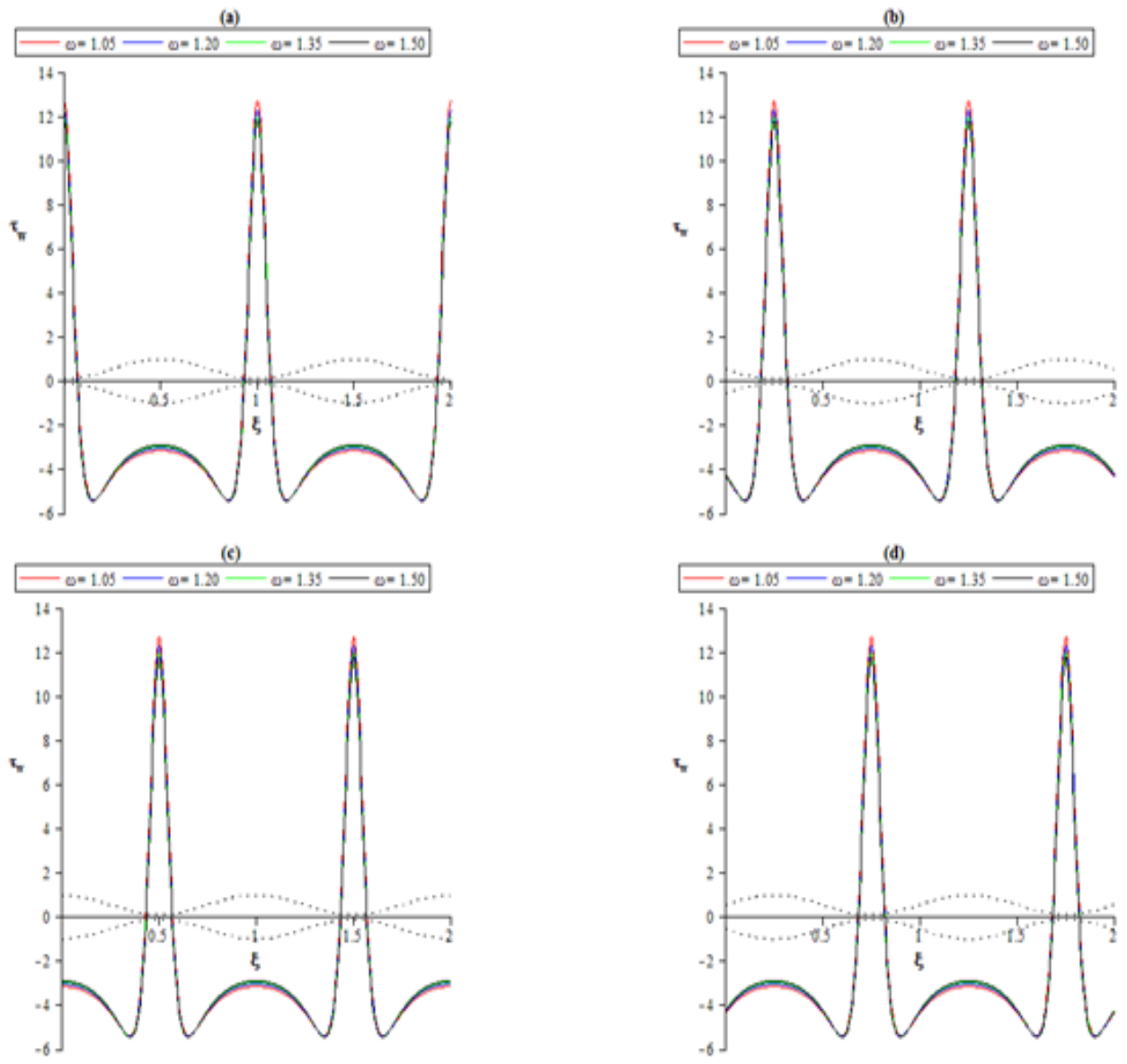

Fig.5. Local wall shear stress vs. axial distance for $\omega=1.05,1.20,1.35,1.50, m=3.0, \phi=0.9, l=2.0$ and various instants (a) $t=0.0,1.0$ (b) $t=0.25$ (c) $t=0.5$ (d) $t=0.75$. Dotted lines represent the position of wave and color solid lines show pressure along the length of channel.
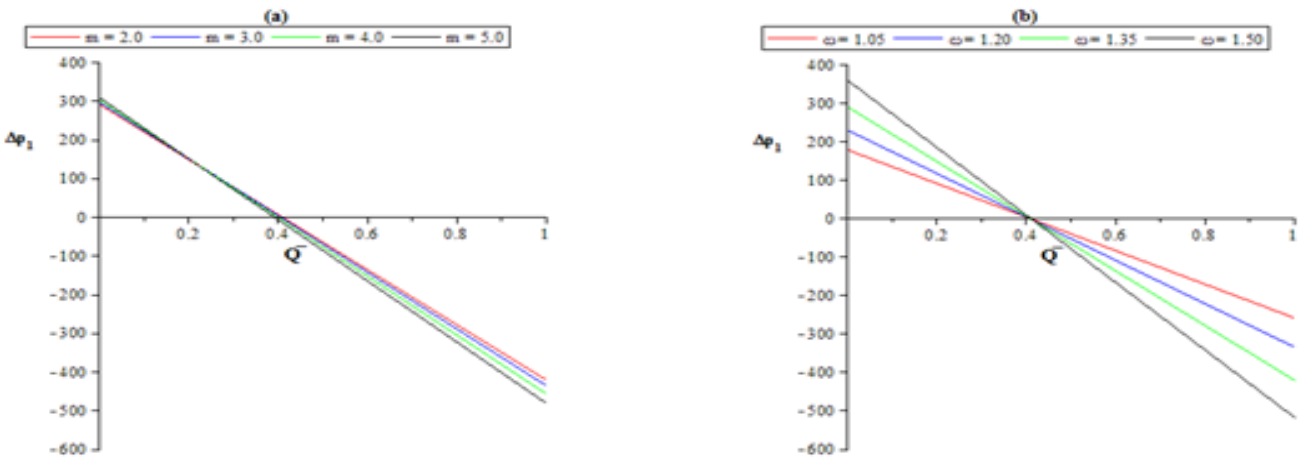

Fig.6. Pressure difference across one wave length vs. average flow rate at $\phi=0.9, t=0.0$.

a) $m=2.0,3.0,4.0,5.0$ and $\omega=1.35$.

b) $\omega=1.05,1.20,1.35,1.50$ and $m=3.0$ 

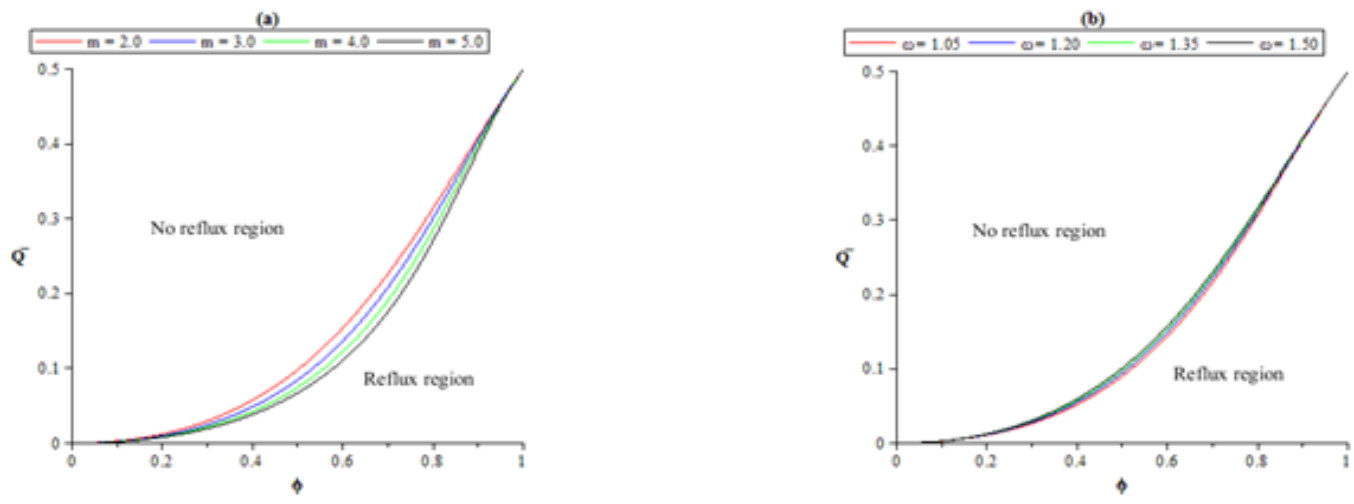

Fig.7. Average flow rate vs. amplitude. Color lines represent reflux limit for $t=0.0$.

a) $m=2.0,3.0,4.0,5.0$ and $\omega=1.35$.

b) $\omega=1.05,1.20,1.35,1.50$ and $m=3.0$.

\section{References}

[1] A.H. Shapiro, M.Y. Jafferin, S.L. Weinberg, Peristaltic pumping with long wave lengths at low Reynolds number, J. Fluid Mech., $35,1969,669-675$.

[2] M. R. Kaimal, Peristaltic pumping of non-Newtonian fluid at low Reynolds number under long wave approximation, J. appl. Mech., $45,1978,32$

[3] Takabatake, K. Ayukawa and A. Mori, Peristaltic pumping in circular cylindrical tubes: a numerical study of fluid transport and its efficiency, J. Fluid Mech., 147, 1988, $267-283$.

[4] V. K. Stud, G. S. Sephon, R. K. Mishra, Pumping action on blood flow by a magnetic field, Bull. Math. Biology, 39, 1977, 385 390.

[5] Kh. S. Mekheimer, Peristaltic flow of blood under effect of a magnetic field in a non-uniform channel, Appl. Math. Comput. , 153, 2004, 763.

[6] N.A.S. Afifi, Aspects of a magneto-fluid with suspended particles, Appl. Math, Infor. Sci., 1, 2007, 103 - 112

[7] N. T. M. Eldabe, A. A. Hassan and Mona A. A. Mohamed, Effect of couple stresses on the MHD of a non-Newtonian unsteady flow between two parallel porous plates, Z.Naturforsch. 58a, 2003, 204- 210.

[8] A. E. Scheidgger, The physics of flow through a porous media, McGraw-Hill, New York, 1963.

[9] E. F. El-Shehawey, K .H. S. Mekheimer, S. F. Kaldas and N. A. S. Afifi, Peristaltic transport through a porous medium, J. Biomath., vol.14, 1999.

[10] E. F. Elshehaway, N. T. Eldabe, E. M. Elghazy and A. Ebaid, Peristaltic transport in an asymmetric channel through a porous medium, Appl. Math. Comput., 182, 2006, $140-150$

[11] C. L. Varshney, The fluctuating flow of a viscous fluid through a porous medium bounded by a porous and horizontal surface, Indian J. pure Apple. Math., 10, 1979, 1558 - 1568.

[12] A. Raptis, C. Peridikis and G. Tzivanidis, Free convection flow through a porous medium bounded by a vertical surface, J. Phys. D: Appl. Phys., 14, 1981, L99 - 108.

[13] A. Raptis, C. Peridikis, Flow of a viscous fluid through a porous medium bounded by a vertical surface, Int. J. Eng. Sci., 21, 1983, No. $11,1327-1336$

[14] N. T. El-Dabe, S. El-Mohandis, Magnetohydrodynamic flow of second order fluid through a porous medium on an inclined porous plane, The Arabian J. for Sci. and Eng., 20, 1995, No. 3, 571 - 580.

[15] N. A. S. Afifi, and N. S. Gad, Interaction of peristaltic flow with pulsatile magneto-fluid through a porous medium, Acta Mech., $149,2001,229-237$.

[16] D. Tripathi, A mathematical model for swallowing of food bolus through the oesophagus under the influence of heat transfer, Int. J.Ther. Sci., 51, 2012, $91-101$.

[17] ] D. Tripathi, Study of transient peristaltic heat flow through a finite porous channel, Math. Comput., Mod. , 57, 2013, 1270 - 1283. 